\title{
A new flavonol from the stem-bark of Premna fulva
}

\section{Guang-ying Chen, ${ }^{\mathrm{a}}$ Chun-yan Dai, ${ }^{\mathrm{a}}$ Tian-shan Wang, ${ }^{\mathrm{a}}$ Cai-wu Jiang, ${ }^{\mathrm{b} *}$ Chang-ri Han, ${ }^{\mathrm{a}}$ and Xiao-ping Song ${ }^{\mathrm{a}}$}

${ }^{a}$ Key Laboratory of Tropical Medicinal Plant Chemistry of Ministry of Education, College of Chemistry \& Chemical Engineering, Hainan Normal University, Haikou, China 571158

${ }^{b}$ School of Pharmacy, Guangxi Traditional Chinese Medical University, Nanning, 530001, P. R.

China

E-mail: cwjiang@126.com

\begin{abstract}
A new flavonol, 4'-hydroxy-8,3'- dimethoxy-6-acroleinylflavan-3,4-diol (1) has been isolated from the ethyl acetate extract of stem-bark of Premna fulva Craib, together with six known compounds, vanillic acid(2), syringaresinol(3), naringenin(4), p-hydroxybenzoic acid (5), apigenin (6) and vitexin (7). The structure of 1 was elucidated on the basis of chemical evidence and intensive spectroscopic analysis including HR-ESI-MS, 1D and 2D-NMR. Compounds 1,3,5,6 and 7 were firstly isolated from this plant. To our regret that compound 1 exhibited no activities towards human cancer cell lines HL-60, BGC-823 and Bel-7402 on the bioassay experiments.
\end{abstract}

Keywords: Premna fulva, Verbenaceae, flavonol

\section{Introduction}

Premna fulva Craib (Verbenaceae), called zhangu in Chinese, is a kind of traditional medicinal plant used widely in the South of China for treatment of lumbago, sprain, rheumarthritis, rheumatoid arthritis and relief of pain in hepatic. The stem bark extract has various biological activities, such as anti-inflammation, analgesic properties, blood-quickening, stasis transforming, protective effect of sciatic nerve and attenuate injuries of soft tissue. ${ }^{1}$ However, most of these studies were focused on the activities and extraction process of the crude extract, ${ }^{2}$ the chemical constituents of this plant had been studied little. Wei song et al had reported the isolation of triterpenoids and flavonoids from the stems of $P$. fulva Craib. ${ }^{3}$ In order to make comprehensive use of this medicinal plant, this paper deals with the systematical chemical constituents from the stem bark of $P$. fulva. A new flavonol, 4'-hydroxy-8,3'-dimethoxy-6-acroleinylflavan-3,4-diol 1, together with six known compounds, vanillic acid 2, syringaresinol 3, naringenin 4, 
$p$-hydroxybenzoic acid 5, apigenin $\mathbf{6}$ and vitexin 7, had been isolated from the ethyl acetate extraction of the stem-bark of $P$. fulva Craib. The structure of $\mathbf{1}$ was elucidated on the basis of chemical evidence and intensive spectroscopic analysis including HR-ESI-MS, 1D and 2D-NMR. Compounds 1,3,5,6 and 7 were firstly isolated from this plant. Compound 1 exhibited weak activities towards human cancer cell lines HL-60, BGC-823 and Bel-7402.

\section{Results and Discussion}

Compounds 1-7 were isolated from the ethyl acetate soluble fraction of the ethanol extract from the stem-bark of $P$. fulva by a combination of several types of chromatography (see Experimental Part). Compounds 2 to 7 were determined by comparison of ${ }^{1} \mathrm{H}-\mathrm{NMR}$ and other physical data with those in the literature. ${ }^{4}$

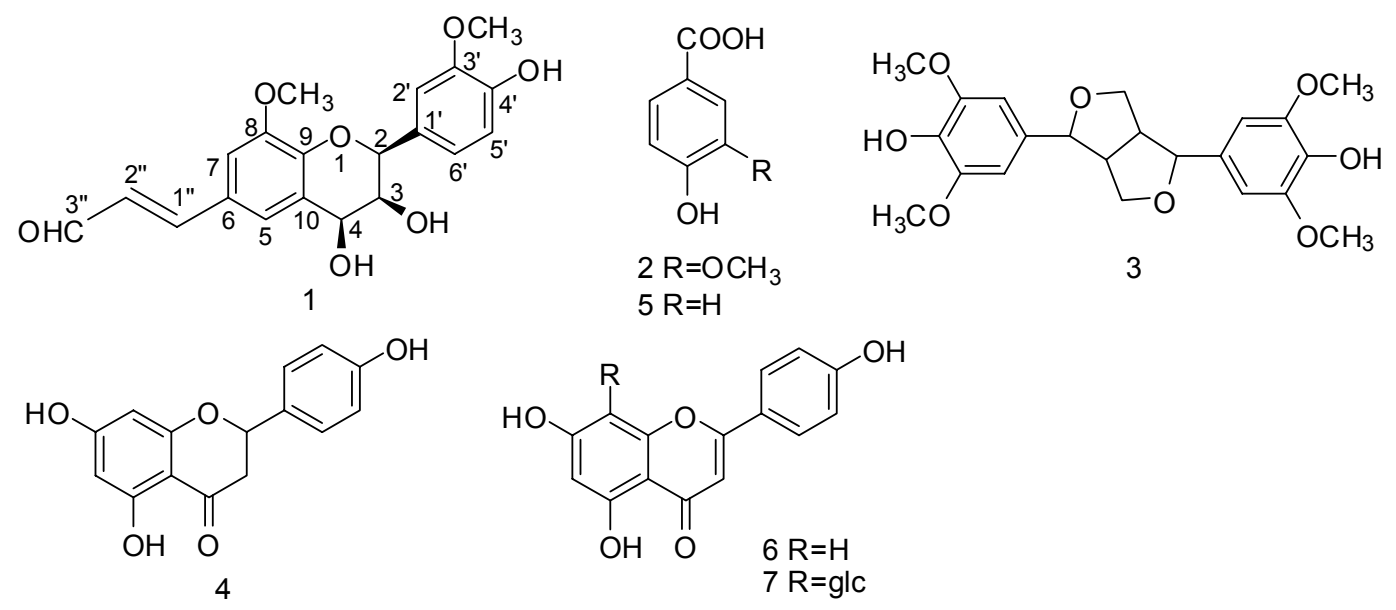

Compound $\mathbf{1}$ was obtained as a pale yellow amorphous powder, which gave a positive $\mathrm{FeCl}_{3}$ test. Its molecular formula was determined as $\mathrm{C}_{20} \mathrm{H}_{20} \mathrm{O}_{7}$ on the basis of negative HR-ESI-MS $\mathrm{m} / \mathrm{z}$ $371.1135[\mathrm{M}-\mathrm{H}]^{-}$, which suggested 11 unsaturated degrees. IR spectrum indicated the presence of hydroxyls $\left(3424 \mathrm{~cm}^{-1}\right)$, carbonyl $\left(1690 \mathrm{~cm}^{-1}\right)$ and the aromatic ring $\left(1596,1500 \mathrm{~cm}^{-1}\right)$, meanwhile strong absorption at 247, 258, 298 and $319 \mathrm{~nm}$ was observed in UV spectrum. The distribution of ${ }^{1} \mathrm{H}-\mathrm{NMR}$ and ${ }^{13} \mathrm{C}$-NMR (Table 1) suggested that $\mathbf{1}$ would be a flavonol skeleton. In ${ }^{1} \mathrm{H}-\mathrm{NMR}$ spectrum, the signals of $7.32(\mathrm{br})$ and $7.29(d, J=1.55 \mathrm{~Hz})$ suggested the existence of one 1,2,3,5-tetrasubstitued benzene ring, the signals of $6.83(d, J=8.24 \mathrm{~Hz}), 6.90(d d, J=1.92,8.24 \mathrm{~Hz})$ and $7.06(d, J=1.92 \mathrm{~Hz})$ suggested the existence of the other 1,3,4-trisubstituted benzene ring, the signals of $9.63(d, J=7.64 \mathrm{~Hz}), 7.58(d, J=15.76 \mathrm{~Hz})$ and $6.66(d d, J=7.60,15.76 \mathrm{~Hz})$ showed the existence of acroloyl moiety with trans formation; meanwhile, three oxygenated methines and two methoxys were also showed in this spectrum.

HSQC spectrum allowed the assignment of all protonated carbons as shown in Table 1, which suggested there were three hydroxyls including one phenolic hydroxyl proton at $7.66 \mathrm{ppm}$ in ${ }^{1} \mathrm{H}-\mathrm{NMR}$ spectrum. Further investigation of HMBC supported the assignment of all the 
quaternary carbons. The structure of 1 was elucidated by ${ }^{1} \mathrm{H}-{ }^{1} \mathrm{H}$ COSY and HMBC correlations using two benzene rings as the starting points.

In HMBC spectrum, H-2 was correlated with C-2' and C-6', H-3 was correlated with C-10 and $\mathrm{H}-4$ was correlated with $\mathrm{C}-5 .{ }^{1} \mathrm{H}-{ }^{1} \mathrm{H}$ COSY spectrum showed those three oxygenated methines were connected together to form a bridge connecting those two benzene rings. While O-1 attaches to the oxygenated aromatic carbon C-9 to form a six-member ring. In the acroloyl moiety, $\mathrm{H}-1$ " shows HMBC correlations to C-5 and C-7, H-2" show HMBC correlation to C-6, suggesting the acroloyl moiety attached to C-6. The attachment sites of those two methoxyl and phenolic hydroxyl also were elucidated by HMBC correlations, which were listed in Table 1.

Table 1. NMR data of 1 (400 MHz for ${ }^{1} \mathrm{H}-\mathrm{NMR}$ and $100 \mathrm{MHz}$ for ${ }^{13} \mathrm{C}-\mathrm{NMR}, \mathrm{CD}_{3} \mathrm{COCD}_{3}$ )

\begin{tabular}{|c|c|c|c|}
\hline Carbons & $\delta_{\mathrm{C}}(\mathrm{ppm})$ & $\delta_{\mathrm{H}}(\mathrm{ppm})$ & HMBC* \\
\hline 2 & 89.4 & $5.66(1 \mathrm{H}, d, J=6.5 \mathrm{~Hz})$ & $3,2^{\prime}, 6^{\prime}$ \\
\hline 3 & 64.3 & $3.63(1 \mathrm{H}, d d, J=12,6.5 \mathrm{~Hz})$ & 10 \\
\hline 4 & 54.2 & $3.91(1 \mathrm{H}, d, J=12 \mathrm{~Hz})$ & 5 \\
\hline 5 & 119.6 & $7.32(1 \mathrm{H}, b r)$ & $7,9,1 "$ \\
\hline 6 & 129.0 & & \\
\hline 7 & 113.5 & $7.29(1 \mathrm{H}, d, J=1.55 \mathrm{~Hz})$ & 5,8 \\
\hline 8 & 145.6 & & \\
\hline 9 & 152.4 & & \\
\hline 10 & 131.2 & & \\
\hline 1' & 133.7 & & \\
\hline $2^{\prime}$ & 110.6 & $7.06(1 \mathrm{H}, d, J=1.92 \mathrm{~Hz})$ & $2,1^{\prime}, 4^{\prime}, 6^{\prime}$ \\
\hline $3^{\prime}$ & 148.4 & & \\
\hline $4^{\prime}$ & 147.5 & & \\
\hline $5^{\prime}$ & 115.7 & $6.83(1 \mathrm{H}, d, J=8.24 \mathrm{~Hz})$ & $1^{\prime}, 3^{\prime}$ \\
\hline $6^{\prime}$ & 119.7 & $\begin{array}{l}6.90(1 \mathrm{H}, d d, J=1.92,8.24 \\
\mathrm{Hz})\end{array}$ & \\
\hline $1 "$ & 154.1 & $7.58(1 \mathrm{H}, \mathrm{d}, J=15.76 \mathrm{~Hz})$ & $7,3^{\prime \prime}$ \\
\hline $2^{\prime \prime}$ & 127.1 & $\begin{array}{l}6.66(1 \mathrm{H}, d d, J=7.70,15.76 \\
\mathrm{Hz})\end{array}$ & 6 \\
\hline 3" & 193.8 & $9.63(1 \mathrm{H}, d, J=7.70 \mathrm{~Hz})$ & \\
\hline $8-\mathrm{OCH}_{3}$ & 56.4 & $3.91(3 \mathrm{H}, s)$ & 8 \\
\hline $3^{\prime}-\mathrm{OCH}_{3}$ & 56.3 & $3.82(3 \mathrm{H}, s)$ & $3^{\prime}$ \\
\hline 4'-OH & & $7.66(1 \mathrm{H}, s)$ & $3^{\prime}, 5^{\prime}$ \\
\hline
\end{tabular}

* Long-range ${ }^{1} \mathrm{H}^{13}{ }^{13} \mathrm{C}$ correlation, protons to carbons.

The stereochemistry of C-2, C-3 and C-4 was determined by NOESY and coupling constants. H-3 and H-4 both lie down the plane with cis configuration with $J_{3,4}=12 \mathrm{~Hz}$ that was strongly 
supported by the coupling constants of the intermediates of leucosyanidin, ${ }^{9}$ and the observed NOE correlation between $\mathrm{H}-2$ and $\mathrm{H}-4$ suggested these two protons were at the same side, which demonstrated all these three protons lie down the plane. It will show small coupling constants if they are located at the upper plane. ${ }^{5}$ The key NOESY correlations were showed in Figure 2, with a stable semi-chair six-membered ring formation. By comparison with the literature data, the relative configuration of 1 can be determined as $2 \mathrm{~S}^{*}, 3 \mathrm{~S}^{*}, 4 \mathrm{~S}^{*}$ with a same C-2 $S$-configuration of those natural products. ${ }^{[10 \mathrm{a}, 10 \mathrm{~b}]}$

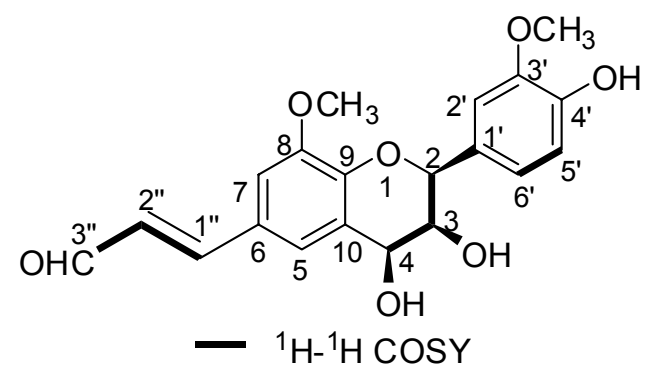

Figure 1. ${ }^{1} \mathrm{H}-{ }^{1} \mathrm{H}$ COSY Correlations for 1.

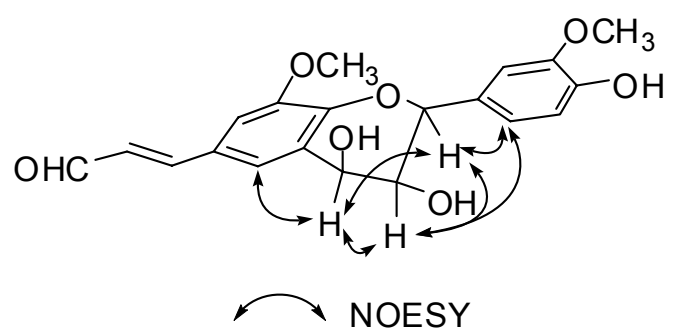

Figure 2. Key NOESY Correlations for 1.

To our knowledge, compound $\mathbf{1}$ is a new flavonol, named premnol, which is similar with the flavonol skeletons of melacacidin and teracacidin. ${ }^{5}$ Melacacidin had the potential inhibition to some animal protein kinases, suggesting that such compounds might act as defensive compounds, acting by interfering with signal transduction in fungal pathogens and herbivores. ${ }^{6}$ Teracacidin and its derivatives were well known for their antioxitive properties. ${ }^{7}$ While, to our interest in the cytotoxic activities, compound $\mathbf{1}$ was evaluated for its cytotoxic activities against human cancer. Unfortunately, compound 1 exhibited no activities towards human cancer cell lines HL-60, BGC-823 and Bel-7402. The antibacterial and fungal activities of compound 1 were yet to be investigated further. 


\section{Experimental Section}

General. IR spectra were run on a Nicolet 5DX-FTIR spectrophotometer ( $\mathrm{KBr}$ plate). UV spectra were recorded on a Shimadzu UV-240 spectrophotometer. NMR spectra were recorded on a Bruker Avance-400 M instrument. ESI-MS were obtained on a Finnigan LCQ Advantage mass spectrometer and HR-ESI-MS on an API Qstar Pulsar-LC/TOF mass spectrometer. Silica gel (200-300 mesh, Qingdao marine Chemical, Qingdao, P. R. China), RP-18 silica gel (50 $\mu \mathrm{m}$, Merck, Darmstadt, Germany) and Pharmadex LH-20 (Amersham Pharmacia Biotech., Hongkong, P. R. China) were used for column chromatography. Precoated silica gel GF254 plates and RP-18 F254 plates (0.25 mm, Merck, Darmstadt, Germany) were used for TLC.

Plant materials. The stem of Premna fulva Craib was collected in July 2004 from Jingxi County, Guangxi Province, P. R. China, and authenticated by Professor Sou-yang Liu (School of Pharmacy, Guangxi Traditional Chinese Medical University, Guangxi Province). A voucher specimen (20040419) was deposited in School of Pharmacy, Guangxi Traditional Chinese Medical University.

Extraction and isolation. The air-dried and powdered stem of $P$. fulva $(15 \mathrm{~kg})$ was extracted with $70 \%$ ethanol. After evaporation of solvents in vacuum, $1.6 \mathrm{~kg}$ residue was obtained. The extract was suspended in $\mathrm{H}_{2} \mathrm{O}(4.0 \mathrm{~L})$ and partitioned successively with petroleum ether $(3 \times 4 \mathrm{~L})$, ethyl acetate $(3 \times 4 \mathrm{~L})$ and $n$ - $\mathrm{BuOH}(3 \times 4 \mathrm{~L})$ to afford the corresponding fractions. The EtOAc extract $(60 \mathrm{~g})$ was subjected to column chromatography $(\mathrm{CC})$ on silica gel eluted with chloroform-methanol gradient to obtained eighteen fractions: Fr. 1 to 18. Fr. 7 (9.42 g) was separated by silica gel CC eluted with chloroform-methanol to provide four subfractions: $F r$. A, B, C, D: $F r$. A was further purified by Sephadex $\mathrm{LH}-20\left(\mathrm{CH}_{3} \mathrm{OH}\right)$ to afford compound $2(25 \mathrm{mg}), \mathrm{Fr}$. $\mathrm{B}$ was separated by silica gel CC eluted with petroleum ether-ethyl acetate to afford compound $\mathbf{3}$ (15 mg), Fr. C was then purified by Sephadex LH-20 $\left(\mathrm{CH}_{3} \mathrm{OH}\right)$ to afford compound $\mathbf{1}(10 \mathrm{mg}), \mathrm{Fr}$. $\mathrm{D}$ was separated by silica gel CC eluted with petroleum ether-ethyl acetate to afford compound 4 $(10 \mathrm{mg}) ; F r .8(6 \mathrm{~g})$ was separated by silica gel CC eluted with petroleum ether-ethyl acetate to afford compound $5(30 \mathrm{mg})$ and $\mathbf{6}(10 \mathrm{mg}) ; F r .14$ was separated by silica gel CC eluted with petroleum ether-ethyl acetate to afford compound $7(5 \mathrm{mg})$.

Cytotoxicity bioassays. The cytotoxicity of compound $\mathbf{1}$ was determined by employing the colorimetric method using ellipticine as reference substance, which was described by Skehan et al. ${ }^{8}$ The reference substance exhibited cytotoxic activity against HL-60, BGC-823 and Bel-7402 cells with $\mathrm{IC}_{50}$ values of $1.45,1.60$, and $0.56 \mathrm{mg} / \mathrm{mL}$, respectively; while it's regretted that compound 1 showed no cytocoxicity.

Premnol (1). A pale-yellow amorphous powder. IR (KBr): 3424, 2938, 2842, 1695, 1596, 1500, 1440, 1160, $1070 \mathrm{~cm}^{-1}$; UV (MeOH) $\lambda_{\max } \mathrm{nm}$ (loge): 216 (1.73), 291 (1.91), 303 (1.96), 334 (1.66); HR-ESI-MS (negative): $\mathrm{m} / \mathrm{z} 371.1135\left([\mathrm{M}-\mathrm{H}]\right.$, calculated for $\left.\mathrm{C}_{20} \mathrm{H}_{19} \mathrm{O}_{7}, 371.1130\right) ;{ }^{1} \mathrm{H}$ and ${ }^{13} \mathrm{C}$ 
NMR see Table 1.

Vanillic acid (2). Colorless needles. m.p. $210-212{ }^{\circ} \mathrm{C} ;{ }^{1} \mathrm{H}-\mathrm{NMR}\left(\mathrm{CDCl}_{3}, 400 \mathrm{MHz}\right) \delta_{\mathrm{H}}: 7.71(1 \mathrm{H}$, $d d, J=8.0,1.2 \mathrm{~Hz}, \mathrm{H}-6), 7.59(1 \mathrm{H}, s, \mathrm{H}-2), 6.97(1 \mathrm{H}, d, J=8.0 \mathrm{~Hz}, \mathrm{H}-5), 3.96\left(3 \mathrm{H}, s, \mathrm{CH}_{3} \mathrm{O}-\right)^{4 \mathrm{a}}$ Syringaresinol (3). Colorless crystals, m.p. 174-176 ${ }^{\circ} \mathrm{C} ;{ }^{1} \mathrm{H}-\mathrm{NMR}\left(\mathrm{CDCl}_{3}, 400 \mathrm{MHz}\right) \delta_{\mathrm{H}}: 6.58$ $\left(4 \mathrm{H}, s, \mathrm{H}-2,6,2^{\prime}, 6^{\prime}\right), 5.57\left(2 \mathrm{H}, b r, \mathrm{OH}-4,4^{\prime}\right), 4.73\left(2 \mathrm{H}, d, J=4.38 \mathrm{~Hz}, \mathrm{H}-7,7^{\prime}\right), 4.28(2 \mathrm{H}, d d, J=$ 9.12,6.90Hz, H-9e,9'e), 3.92 (2H, m, H-9a,9'a), 3.89 (3H, s, MeO-4), 3.09 (2H, m, H-8,8').

${ }^{13} \mathrm{C}-\mathrm{NMR}\left(\mathrm{CDCl}_{3}, 100 \mathrm{MHz}\right) \delta_{\mathrm{C}}: 147.11\left(\mathrm{C}-3,5,3^{\prime}, 5^{\prime}\right), 134.26\left(\mathrm{C}-4,4^{\prime}\right), 132.01\left(\mathrm{C}-1,1^{\prime}\right), 102.67$ $\left(\mathrm{C}-2,6,2^{\prime}, 6^{\prime}\right), 85.98\left(\mathrm{C}-7,7^{\prime}\right), 71.72\left(\mathrm{C}-9,9^{\prime}\right), 56.30(\mathrm{OMe}), 54.25\left(\mathrm{C}-8,8^{\prime}\right){ }^{4 \mathrm{~b}}$

Naringenin (4). Colorless crystals, m.p. 243-244 ${ }^{\circ} \mathrm{C}$; ${ }^{1} \mathrm{H}-\mathrm{NMR}\left(\mathrm{CD}_{3} \mathrm{COCD}_{3}, 400 \mathrm{MHz}\right) 12.18$ (1H, $s, \mathrm{OH}-5), 9.57$ (1H, $s, \mathrm{OH}-7), 8.5$ (1H, $\left.s, \mathrm{OH}-4^{\prime}\right), 7.39\left(2 \mathrm{H}, d d, J=8.49,1.96 \mathrm{~Hz}, \mathrm{H}-2^{\prime}, 6^{\prime}\right)$, $6.90\left(2 \mathrm{H}, d d, J=8.62,2.10 \mathrm{~Hz}, \mathrm{H}-3^{\prime}, 5^{\prime}\right), 5.96(1 \mathrm{H}, d, J=2.23 \mathrm{~Hz}, \mathrm{H}-8), 5.95(1 \mathrm{H}, d, J=2.23 \mathrm{~Hz}$, H-6), $5.45(1 \mathrm{H}, d d, J=12.87,3 \mathrm{~Hz}, \mathrm{H}-2,3.17(1 \mathrm{H}, d d, J=17.13,12.87 \mathrm{~Hz}, \mathrm{H}-3 \mathrm{e}), 2.73(1 \mathrm{H}, d d, J$ $=17.13,2.99 \mathrm{~Hz}, \mathrm{H}-3 \mathrm{a}){ }^{4 \mathrm{c}}$

p-Hydroxybenzoic acid (5). Colorless crystals, m.p. 149-151 ${ }^{\circ} \mathrm{C} ;{ }^{1} \mathrm{H}-\mathrm{NMR}\left(\mathrm{CD}_{3} \mathrm{OD}, 400 \mathrm{MHz}\right) \delta$ : $7.86(2 \mathrm{H}, d d, J=8.75,1.96 \mathrm{~Hz}, \mathrm{H}-2,6), 6.81(2 \mathrm{H}, d d, J=8.78,1.92 \mathrm{~Hz}, \mathrm{H}-3,5)$.

${ }^{13} \mathrm{C}-\mathrm{NMR}\left(\mathrm{CD}_{3} \mathrm{OD}, 100 \mathrm{MHz}\right) \delta_{\mathrm{C}}: 170.18(\mathrm{COOH}), 163.35(\mathrm{C}-4), 133.02(\mathrm{C}-2,6), 122.78(\mathrm{C}-1)$, $116.04(\mathrm{C}-3,5){ }^{4 \mathrm{~d}}$

Apigenin (6). Yellow power $m p$ 343 344 ${ }^{\circ} \mathrm{C} ;{ }^{1} \mathrm{H}-\mathrm{NMR}\left(\mathrm{C}_{5} \mathrm{D}_{5} \mathrm{~N}, 400 \mathrm{MHz}\right) \delta_{\mathrm{H}}$ : $13.75(1 \mathrm{H}, s$, 5-OH), 7.90(2H, $\left.d, J=8.4 \mathrm{~Hz}, \mathrm{H}-2^{\prime}, 6^{\prime}\right), 7.19\left(2 \mathrm{H}, d, J=8.4 \mathrm{~Hz}, \mathrm{H}-3^{\prime}, 5^{\prime}\right), 6.89(1 \mathrm{H}, s, \mathrm{H}-3), 6.79$ $(1 \mathrm{H}, d, J=2 \mathrm{~Hz}, \mathrm{H}-8), 6.73(1 \mathrm{H}, d, J=1.6 \mathrm{~Hz}, \mathrm{H}-6){ }^{4 \mathrm{e}}$

Vitexin (7). yellow power, $m p$ 260 262 ${ }^{\circ} \mathrm{C}$; ${ }^{1} \mathrm{H}-\mathrm{NMR}$ (DMSO-d 6 , $\left.400 \mathrm{MHz}\right) \delta_{\mathrm{H}}$ : $13.17(1 \mathrm{H}, s$, OH-5), 10.78 (1H, s, OH-7), 10.35 (1H, $\left.s, \mathrm{OH}-4^{\prime}\right), 8.02\left(2 \mathrm{H}, d, J=8.8 \mathrm{~Hz}, \mathrm{H}-2^{\prime}, 6^{\prime}\right), 6.89$ (2H, $d, J$ $\left.=8.8 \mathrm{~Hz}, \mathrm{H}-3^{\prime}, 5^{\prime}\right), 6.78(1 \mathrm{H}, s, \mathrm{H}-6), 6.27(1 \mathrm{H}, s, \mathrm{H}-3), 4.99(m, \mathrm{OH}-\mathrm{Glu}), 4.68(1 \mathrm{H}, d, J=10 \mathrm{~Hz}$, $\left.\mathrm{H}-1^{\prime \prime}\right), 3.24 \sim 3.87\left(6 \mathrm{H}, m, \mathrm{H}-2^{\prime \prime} \sim 6^{\prime \prime}\right){ }^{4 \mathrm{f}}$

\section{Acknowledgements}

This work was supported by the National Natural Science Foundation of China (20862005), the Key Project of the Chinese Ministry of Education (No.207090) and the Natural Science Foundation of Hainan Province, China (No.070207).

\section{References}

1. Wang, J. W. Plants 1989, 1, 28.

2. (a) Chen, L. F.; Lin, C.W.; Wei, T. Y.; Wei, W. X.; Chen, H. Y. Jingxi Huagong 2007, 24, 355. (b) Zhang, G.H.; Jing, S. H.; Pan, H. P.; Lu, M.; Chen, X. Y.; Rong, Y. P. Zhongguo Yaofang 2006, 17, 590. (c) Li, X. Y.; Luo, W. Q.; Yang, L. F.; Pan, H. P. Guangxi Minzu Xueyuan Xuebao (Ziran Kexueban) 2005, 11, 121. 
3. (a) Wei, S.; Si, S. L.; Xu, X. J.; Pu, Q. L.; Pannell, L. K.; Highet, R. J. Planta Medica 1991, 57, 93. (b) Zeng, Q.; Liu, C. J.; Liu, L. G. Zhongguo Yaoke Daxue Xuebao 1989, 20, 94. (c) Dai, C. Y.; Chen, G. Y.; Zhu, G. Y.; Fang, H. X.; Jiang, C. W. Zhongcaoyao 2007, 38, 34.

4. (a) Chen L.; Du L. J.; Ding Y.; Xing D. M.; Wang W. China Journal of Chinese Materia Medica 2005, 30, 1340. (b) Gu H. F.; Chen R. Y.; Sun Y. H.; Liu F. China Journal of Chinese Materia Medica 2004, 29, 232. (c) Feng Y. L.; XU L. Z.; Yang S. L.; Zou Z. M. Chinese Traditional and Herbal Drugs 2005, 36, 1610. (d) Qiu Y. K.; Dou D. Q.; Pei Y. P.; Yoshikawa M; Matruda H; Chen Y. J. China Journal of Chinese Materia Medica 2005, 30, 1824. (e) Nan H. H.; Zhang S.; Wu J. Chinese Traditional and Herbal Drugs 2005, 36, 492. (f) Chen Q.; Wu L. J.; Ruan L. J. Journal of Shenyang Pharmaceutical University 2002, 19, 257.

5. Pietarinen, S. P.; Willför, S. M.; Sjöholm, R. E.; Holmbom, B. R.. Holzforschung. 2005, 59, 94.

6. Polya, G.M. ; Foo, L.Y.. Phytochemistry. 1994, 35, 1399.

7. Tachi, M.; Tange, J.; Nagadomi, W.; Suzuki, Y.; Terashima, N.; Yasuda, S.. Acacia mangium. Mokuzai Gakkaishi. 1989, 35, 731.

8. Skehan, P.; Storeng, R.; Scudiero, D.; Monks, A.; McMahon, J.; Vistica, D.; Warren, J. T.; Bokesch, H.; Kenny, S.; Boyd, M. R. Journal of the National Cancer Institute 1990, 82, 1107.

9. Kikuchi T.; Nishimura M.; Hoshino A.; Morita Y.; Iida S.; Saito N.;Honda T. Heterocycles 2003, 60, 1469.

10. (a) Sha N.; Guan S. H.; Lu Z. Q.; Chen G. T.; Huang H. L.; Xie F. B.; Yue Q. X.; Liu X.; Guo D. A. Journal of Natural products 2009, 72, 799. (b) Agust A.; Maehara S.; Ohashi K.; Simanjuntak P.; Shibuya H. Chemical \& Pharmaceutical Bulletin 2005, 53, 1565 